\title{
QoS based Vertical Handoff Algorithm for Next Generation of Wireless Network
}

\author{
Ravindra C. Sanap \\ Dept. of Computer Engg. \\ G.H. Raisoni College of Engg. \& \\ Mngt. Ahmednagar, India
}

\author{
S.S. Sambare \\ Dept. of Computer Engg. Pimpri \\ Chinchwad College of Engg. Pune, \\ India
}

\begin{abstract}
In this paper present the different issue of vertical handoffs in heterogeneous wireless networks. The uses of wireless communications are popular and increasing its demand over worldwide. In the world every one likes to be connected seamlessly any time any where through the best network and good service. The proposed system its aim is that it provides better service to the user. In this paper consider the different parameters like available bandwidth of WLAN or WiMAX, remaining Power of node, high level security of node and cost of node. From above parameters current status of networks and detected node information is collected and based on it Vertical Handoff algorithm takes decision. Handoff decision will take place only when network cannot satisfy the QoS requirement.
\end{abstract}

\section{General Terms}

VHOM (Vertical Handoff Manager Algorithm)

\section{Keywords}

QoS, Vertical Handoff, VHOM, Wi-Fi, WLAN, WiMAX.

\section{INTRODUCTION}

Always connected it means where a person can choose the best available access networks and devices at any point of time as in [1]. A very popular standard is IEEE802.11 because it support to Wireless LANs (WLAN), and it widely used in broadband wireless access network, because its cost is minimum and also it support to high capacity. There is another standards family is IEEE 802.16, it support to emerging broadband wireless access technique, World- wide Interoperability for Microwave Access (WiMAX), WiMAX having its high bandwidth and it also support to long-range transmission with QoS parameters.

\subsection{Architecture of WLAN}

A typical WLAN network consists of two entities: APs and STAs. APs are the Base Stations for a wireless network. They communicate wireless with STAs by radio waves. STAs are wireless clients which connect to the APs in getting access to the network. The Lightweight and Autonomous architectures are used in WLAN environment. Each of the architectures has wide impact on wired LAN architecture. The choosing of WLAN architecture is based on the consideration of building, future proof, integrated wired. Both architectures are popular but Lightweight architecture has plus advantages over the WLAN market.

\subsubsection{Lightweight Model}

The Lightweight model is the part of WLAN architecture. In this architecture the Lightweight model is very simple shown Fig 1.; the devices that provide the communication to the end user as Access Layer are identified by lightweight. In lightweight model the Distribution layer provides inter communication and the core layer (top layer) is responsible fast and consistent data between networks. Wireless Access Point (WAP) resides at the interface of access layer and provides the communication interface to end user [19].

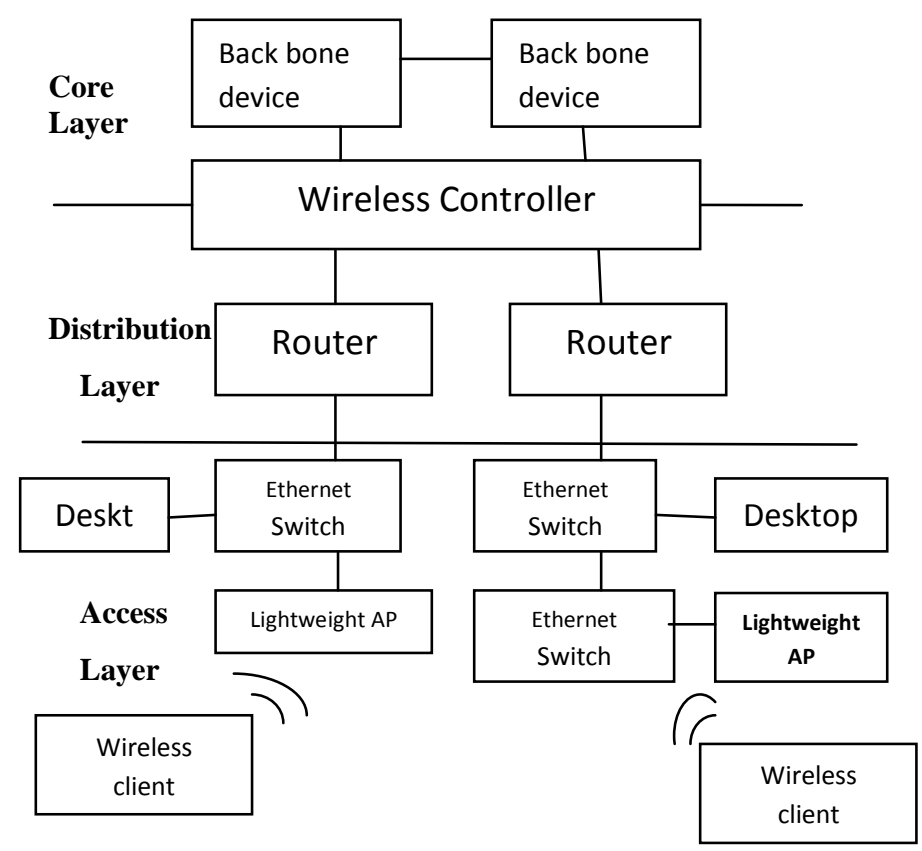

Fig 1 Lightweight Architecture Model

\subsubsection{Autonomous Model}

Autonomous Wireless Access Point sustains the switching and strong security as well as networking function that are indispensable to route the wireless traffic. As in autonomous system there is no concept of the visibility of WAP so it cannot make the load balancing.

Autonomous model cannot differentiate whether nearest WAP is part of WLAN infrastructure or illegal rouge WAP. The difference between the autonomous and lightweight is negligible. The difference is only this that lightweight have one extra component (WLAN controller) [20]. The Autonomous model shown as Fig 2. 


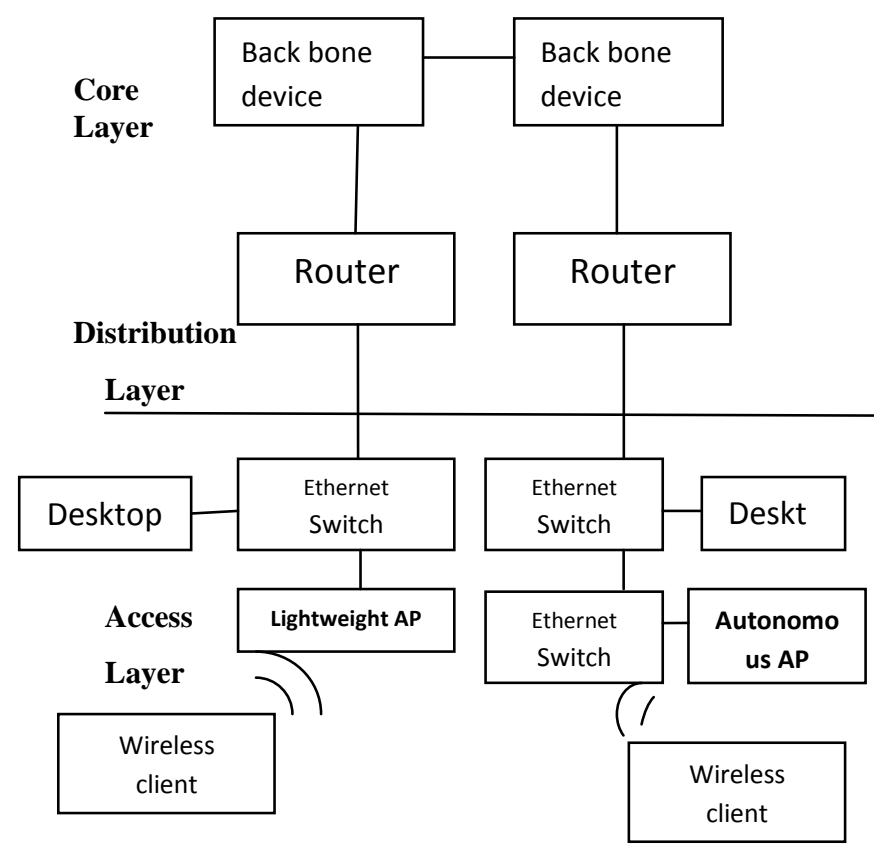

Fig 2 Autonomous Architecture Model

\subsection{WiMAX Standard}

Worldwide Interoperability for Microwave Access. The fiber optic transport services providing the high bandwidth and data rates is replaced by WiMAX wireless technology all across the world. WiMAX is emerging technology to fulfill the high data rate and also provides QoS requirements of the customers, it is the cheap for deployment of voice services with no need of line of sight wireless channel. WiMAX support to electromagnetic waves, so it support of adoptive coding and different operation modes, because of electromagnetic waves voice and data services can easily be transported by WiMAX network platform.

The WiMAX Network Architecture defines a framework consisting of several functional entities and interconnections. Fig 3. Shows this framework in simplified manner, followed by a description of each entity. WiMAX is an IP based, wireless broadband access technology that provides performance of similar to $802.11 / \mathrm{Wi}-\mathrm{Fi}$ networks technology with the good coverage and QOS (quality of service) of cellular networks. It also Provide fixed, nomadic, portable and eventually mobile wireless broadband without the need for direct LOS to base station. WiMAX revision provides up to $40 \mathrm{Mbps}$ data rate in typical 3-10 $\mathrm{km}$ base station radius.

\subsubsection{WiMAX Network Architecture}

The WiMAX architecture as shown in Fig 1.3 and the details architecture as summarize are as follows.

\section{NAP: Network Access Provider}

A business entity that provides WiMAX radio infrastructure.

NSP: Network Service Provider Just like the NAP, the NSP is a business entity. It provides IP connectivity and WiMAX services. The level of services is legally binded through contractual agreements with one or more NAPs.

\section{SS/MS: Subscriber Station/Mobile Station}

SS/MS is an entity that wants to make a connect to the WiMAX network technology.

ASN: Access Service Network
This is the point of entry for the SS/MS into the WiMAX network. ASN entity must support for set of functions required to connect a client to the network i.e. authorization, authentication, session management, IP-address allocation, network discovery, QoS etc.

\section{CSN: Connectivity Service Network}

The CSN is the part of the network which provides IP connectivity services. It consists typically of routers, servers, proxies, and gateways etc. It also providing functions like Internet access and peer-to-peer services.

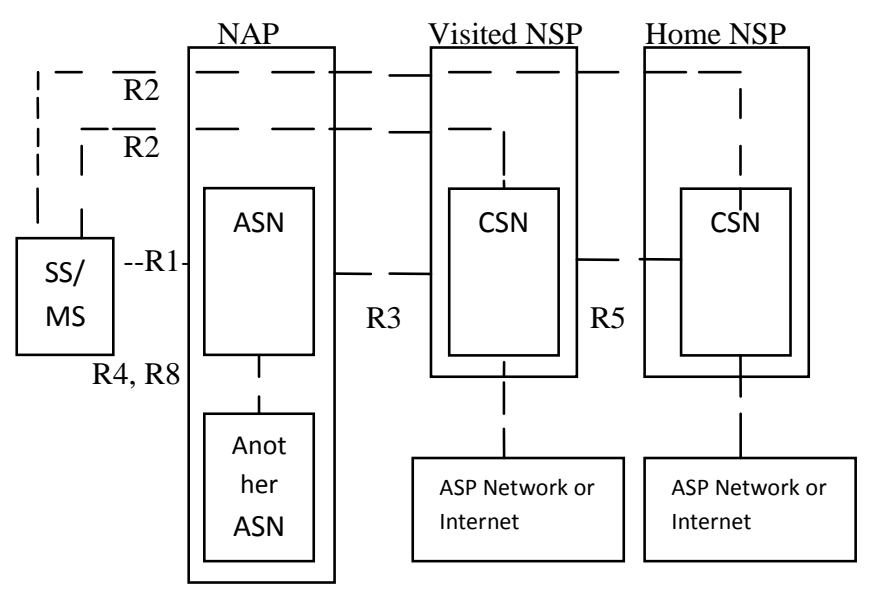

Fig 3 WiMAX Network Architecture

In this paper, to design effective handoff technique to satisfy the fully QoS parameters. The major work of this paper can be summarized as below.

Network detection: A VHO management (VHOM) scheme to take VHO decisions in different (WLANs and WiMAX) networks when each station monitors the quality of connections and detects the network conditions. VHOM always selects the best network having better service to serve station rather than a preferred network.

Bandwidth estimation: By considering QoS parameters to make a handoff decision, two different bandwidth estimation algorithms has been given in existing system to find out the available bandwidth of WLAN and WiMAX networks[6][17].

Power: In this, at the time taking handoff decision if node having less power then there will be loss of call, so design this technique in a such way that it calculates power of nearest neighboring node and based on it take decision.

The Security: The risks are major issue in any wireless technology and some of these risks are similar to wired networks. For some applications, confidentiality of data, communication or integrity of data transmission may be difficult issue. So that those network node having high security level may be considered over other network having lower security level. Therefore, security is one of the main parameter in decision making.

Network Cost: The Cost of various services is varies according to their variety plans, so cost is a big problem. Each network have a different policy of cost, Based on the interworking architecture of WLAN and WiMAX, in order to enable stations to intelligently monitor the quality of current connections and based on it make an accurate handoff decision. So in some cases the cost of network should be 
taken into account for taking handoff decisions. In proposed system assigned different network cost to different node. Therefore, those nodes having minimum cost that will be selected during vertical handoff decision.

\section{PROPOSED VHO MANAGEMENT SCHEME}

In this paper design a VHOM scheme, this works on the MAC layer protocols of IEEE 802.11 and IEEE 802.16 standards. The major functions of the proposed VHOM scheme include traffic measurement, network status detection, power consumption, security level, cost of network, handoff decision, and connection transition. The functions are performed by four modules as shown in Fig 4

\subsubsection{Handoff decision module (HDM)}

This is the third module of VHOM designed system which is the core component of VHOM. It gathers information from other modules and manipulates their operation. It decides the need to launch a handoff decision process and selects the target network based on the available handoff policies.

\subsubsection{Connection transition module (CTM)}

This is the last module of VHOM system, which transfers all the connections of the station from the service network to the selected target network.

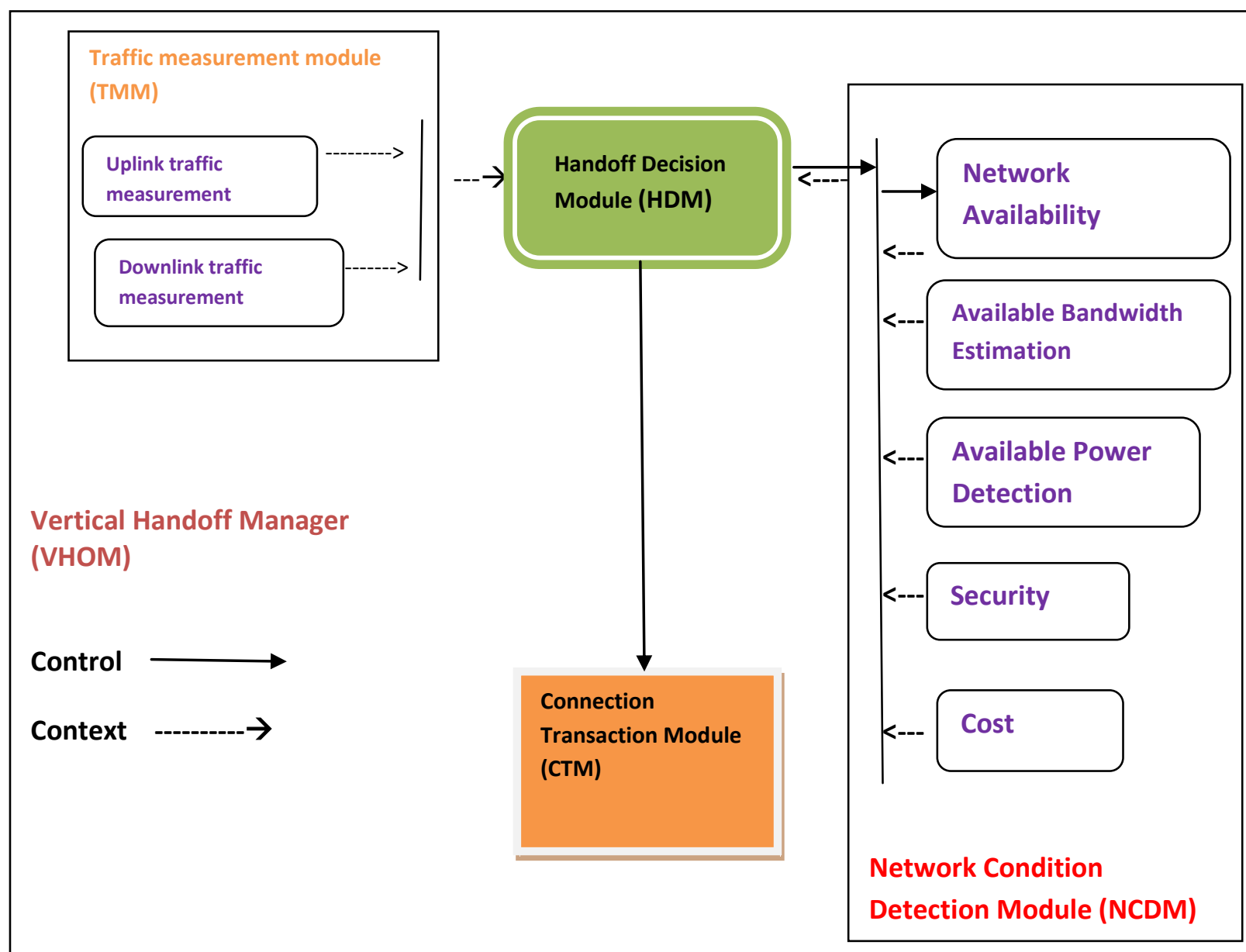

\subsection{Modules of Proposed system}

\subsubsection{Traffic measurement module (TMM)}

This is the first module of VHOM designed system, which periodically measures the throughput of the network and provides reports to the Handoff decision module (HDM).

\subsubsection{Network condition detection module (NCDM)}

This is the second module of VHOM designed system, which detects the availability of possible networks and estimates the performance of the available network. indicate the throughput of the uplink or downlink connections cannot satisfy the QoS requirement of the traffic at the station, it will initiate the NCDM to detect the performance of the other network. The availability of the network will be detected first. If the other network cannot be found, HDM will terminate this handoff attempt and restart after a period if the measured traffic still cannot meet QoS requirement. Otherwise, if the network can be detected, NCDM will awaken the corresponding MAC layer from the sleep mode to estimate the bandwidth of the detected network domain using bandwidth estimation algorithms [17].

After estimating available bandwidth, search for neighboring nodes having enough power. Subsequently, the estimated reports will be submitted to HDM to make a decision on 
handoff. If the estimated available bandwidth can satisfy the QoS requirement of the traffic at the station, HDM will decide the new network domain as the target network and initiate CTM to perform a handoff operation. Otherwise, NCDM will be started to evaluate the bandwidth again after a period of time. Once CTM receives a command to launch a handoff, it will issue a gratuitous Address Resolution Protocol (ARP) message to notify the binding of the IP address and the MAC address corresponding to the target network. Hereafter, the data destined to the station will be transmitted by access gateway via the target network. While the IP address is not changed, the VHO process is transparent to upper layers. When making a handoff decision, it should be noticed that the resource allocation approach in WiMAX systems is different from that in WLANs.

In WLANs, the medium is shared by multiple stations in a contention manner. And only the total available bandwidth can be estimated. However, the wireless resource in WiMAX networks is scheduled by BS and is partitioned into downlink and uplink sections. Then the available bandwidth should be estimated for downlink and uplink directions, respectively. Therefore, the handoff direction should be considered when making a VHO decision.

\section{BANDWIDTH ESTIMATION AND PROPOSED POWER SAVING, SECURITY AND COST MODULE}

\subsection{Bandwidth Estimation in WLANs}

The IEEE 802.11 MAC of access method is Distributed Coordination Function (DCF) known as carrier sense multiple access with collision avoidance (CSMA/CA) [7]. Carrier sense multiple access method performed by the advertising reservation before sending data. The exchange of request containing send/clear (RTS/CTS) frames prior to the data transmission, the source station send request to send (RTS) request to destination station and the destination station replies with clear to send (CTS). Remaining stations that hear RTS/CTS and update their NAV to be busy state for a period of time given in the RTS/CTS frames.

Then the average utilization of the medium can be calculated by using following formula

$$
A_{U t i l-m e d}(t-\alpha, t)=\frac{S_{\text {data }}(t-\alpha, t)}{\alpha}
$$

The reserved resource that can be used for data transmission with the help of following

$$
\begin{aligned}
& R_{\text {resource }} \\
& =\frac{\mu_{\text {data }}}{\mu_{\text {data }}+\mu_{R / C / A}+\left(3 T_{\text {SIFS }}+T_{D I F S}+T_{\text {backoff }}(t) C(t) / 8\right)}
\end{aligned}
$$

Where $\mu_{\text {data }}$ is the mean data packet size in bytes of the desired station, $\mu_{R / C / A}$ is the sum of RTS/CTS/ACK frames size, TSIFS and TDIFS are time durations of SIFS and DIFS. $T_{\text {backoff }}(t)$ Containing denote the average random backoff time, and $\mathrm{C}(\mathrm{t})$ is the data rate at time $\mathrm{t}$.

Based on the above average utilization and reserved resource information, available bandwidth of WLAN can be calculated as below

$$
B W_{\text {available }}(t)=R_{\text {resource }}-A_{U t i l-m e d}(t-\alpha, t) C(t)
$$

\subsection{Bandwidth Estimation in WiMAX Systems}

The frame consists of a down link subframe and an uplink subframe divided in IEEE 802.16 protocol. The data bursts are used for allocate bandwidth resources. And the burst containing an integer number of physical slots (PSs) and how many demand of resource as well as what type of QoS parameters required, BS determines the bandwidth that a station can obtain in one frame for receiving downlink data or transmitting uplink data respectively, and then broadcasts this allocation information through information element (IE) in the DL-MAP and UL-MAP messages at the beginning of each frame [8]. Then the number of unused PSs of this period is equal

$$
\begin{aligned}
& \mathrm{PS}_{\mathrm{UL}_{\_} \text {freeslot }}(\mathrm{t}-\boldsymbol{\beta}, \mathrm{t})=\mathbf{n P S} S_{\mathrm{UL}_{\_} \text {total }}-\sum_{\mathrm{i}=1}^{\mathrm{n}} \mathrm{PS}_{\mathrm{UL} \_ \text {used }} \\
& \text { PS }_{\text {DL_freeslot }}(t-\beta, t)=n P S_{D_{-} \text {total }}-\sum_{i=1}^{n} P S_{\text {DL_used }}
\end{aligned}
$$

Where PSUL-used(4) and PSDL-used(5) denote the utilized PSs in the ith downlink and uplink sub frame.

Based on above, the available bandwidth can be estimated by the following expression

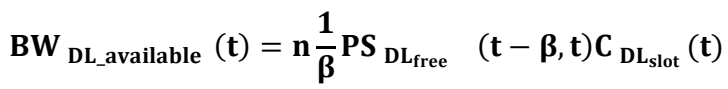

$$
\begin{aligned}
& B_{\text {UL_available }}(t)=n \frac{1}{\beta} P S_{U L_{\text {free }}}(t-\beta, t) C_{U L_{\text {slot }}}(t)
\end{aligned}
$$

Where CDL-slot $(\mathrm{t})$ and CUL-slot $(\mathrm{t})$ are the number of bits that can be transmitted in one downlink or uplink PS. The values depend on the modulation and coding schemes (MCS) used at the physical layer.

\subsection{Proposed Power Saving Module}

Wireless devices running on battery, so they have limited power consumption. If the battery level decreases, switching for a network to another network with low power consumption can provide a longer usage time. For example, if a device with the battery almost exhausted, switching from a WLAN to a WiMAX network would be a smart decision. This is because, when they operate in a WiMAX network, the device is inactive for an extended period of time.

The Power requirements become a critical issue if the hand held battery is low. In such situations, it is preferably transferred to an attachment point, and this will extend battery life [9].

So before doing handoff first find out list of neighboring nodes and will check there remaining power. If it will be below threshold value i.e. $10 \%$ of total energy, then set flag to 1 otherwise to 0 . If flag set at neighboring node is 0 then avoid switching to that particular network otherwise initiate handoff.

The remaining energy of the node can be expressed as: 


$$
N_{\text {Remaining_energy }}=N_{\text {Initial_energy }}-N_{\text {Consumed_energy }}
$$

Where, $\boldsymbol{N}_{\text {Remaining_energy }}$ means remaining energy of node, $\boldsymbol{N}_{\text {Initial_energy }}$, is Node Initial energy and $\boldsymbol{N}_{\text {Consumed_energy }}$ is Consumed energy of node. The Consumed energy can be defined as below

$$
\begin{aligned}
N_{\text {Consumed_energy }} & =\text { RecN }_{\text {Initial_packets }} * \operatorname{Rec}_{\text {energy }} \\
& + \text { Trans }_{\text {packets Trans }} \text { Trengy }
\end{aligned}
$$

Where, $\boldsymbol{R e c}_{\text {Initial_packets }}$ is received packets, $\boldsymbol{R e c}_{\text {energy }}$ Receiving power, Trans packets $_{\text {is }}$ Transmitted packets and Trans $_{\text {energy }}$ is Transmission power.

\subsection{Proposed Security Module}

With the increasing demand of wireless networks, seamless and secure handoff has become an important factor in wireless networks. The network security consists of the provisions and policies adopted by the network to prevent and monitor unauthorized access, misuse, modification, and network accessible resources. In a wireless environment, data is broadcast through the air and people do not have physical controls over the boundaries of transmissions. The security features provided in some wireless products may be weaker; to attain the highest levels of integrity, authentication, and confidentiality, network security features should be embedded in the handoff policies.

The proposed system have set security level to every nodes randomly, Some nodes having high level(1)and other having low level(0). There is also provided additional functionality to node level like to encrypt data at sender side and decrypt data receiver side for protecting the data over insecure network. The HDM module always gives first priority to select highest level (Level=1) of security parameter of nodes as compare to lowest level at the time of handoff decision. In some case if there is only one node available then proposed HDM always select it first in that case it check the security level.

\subsection{Proposed Cost Module}

A multi criteria algorithm for handoff should also consider the network cost factor. The cost is to be minimized during VHO in wireless networks. The new call arrival rates and handoff call arrival rates can be analyzed using cost function. Next Generation heterogeneous networks can combine their respective advantages on coverage and data rates, offering a high Quality of Service (QoS) to mobile users. Therefore, network selection cost is important in handoff decisions. In proposed scheme have set cost to nodes those nodes have less that will take at the time of handoff decision.

Let $\mathrm{CBT}$ be expressed as $\mathrm{C}_{\mathrm{BT}}=\left\{\left(\mathrm{BS}_{1}, \mathrm{BS}_{2} \ldots \ldots . \mathrm{BS}_{\mathrm{n}}\right)\right\} \mathrm{n} \leq \mathrm{M}$ is the target $\mathrm{BS}$ is selected from set $\mathrm{C}_{\mathrm{BT}}$ for the least cost as follows

$$
\begin{array}{r}
\min _{\mathbf{n}}\left\{\left(\mathbf{R}_{\mathbf{u}}+\mathbf{R}_{\mathbf{d}}\right) * \mathbf{T}_{\mathbf{c}}^{\mathbf{n}} * \mathbf{C}_{\mathbf{n}} \quad\right\} \forall \mathbf{B S}_{\mathbf{n}} \varepsilon \mathbf{C}_{\mathbf{B T}}, \mathbf{R}_{\mathbf{d}} \\
\leq \mathbf{B}_{\mathbf{d}}^{\mathbf{n}}, \mathbf{R}_{\mathbf{u}} \leq \mathbf{B}_{\mathbf{u}}^{\mathbf{n}} \quad \mathbf{1} \leq \mathbf{n} \leq \mathbf{N}
\end{array}
$$

Where $\mathrm{Rd}$ and $\mathrm{Ru}$ are the MN's desired downlink and uplink data rates, $\mathbf{C}_{\mathbf{n}}, \mathrm{B}_{\mathrm{d}}^{\mathrm{n}}$ and $\mathrm{B}_{\mathrm{u}}^{\mathrm{n}}$ are BSn's data cost, available downlink bandwidth and available uplink bandwidth, respectively.

\section{THE ALGORITHM OPERATION OF THE PROPOSED VHOM SCHEME}

\subsection{Algorithm}

1 Check if the QoS requirements are satisfied or not.

2 If satisfied

2.1 Stay with current network

3 Else

3.1 Detect the performance of the other network

3.2 If the network can be detected

3.2.1 Estimate the bandwidth of the detected network domain

3.2.2 If estimated available bandwidth can satisfy the QoS

3.2.2.1 If neighbouring nodes having enough power

3.2.2.2 If nodes having high security

3.2.2.3 If nodes having low cost

3.2.2.4. Perform a handoff operation.

3.2.2.5 Else

3.2.2.2.1 Go to step 3.1

\subsubsection{Else}

3.2.3.1 Evaluate the bandwidth again after a period of time.

\subsection{Else}

3.3.1 Terminate this handoff attempt and restart after a period if the measured traffic still cannot meet QoS requirement.

\section{RESULTS}

Graphs are plotted for proposed work and existing method. In the existing method handoff were done based on available bandwidth factor were as in proposed system handoff decision is done based on available bandwidth, power consumption, security and cost parameters. By comparing existing method and proposed method considering different parameters. The simulation parameters as shown in table 1.1.

Table 1.1: Simulation parameters for Vertical Handoff Evaluation

\begin{tabular}{|l|c|}
\hline Simulation Parameter & Values \\
\hline Standards & $802.11 \mathrm{~b}, 802.16$ \\
\hline Packet Interval & $0.1 \mathrm{~ms}$ \\
\hline Initial Energy & $100 \mathrm{jule}$ \\
\hline Channel Bandwidth & $40 \mathrm{MHz}$ \\
\hline
\end{tabular}




\begin{tabular}{|l|c|}
\hline Data rate of WLAN & $11 \mathrm{Mb}$ \\
\hline Data rate of WiMAX & $40 \mathrm{Mb}$ \\
\hline No of Base station & 01 \\
\hline No. of Access point & 01 \\
\hline No. of Wired node & 01 \\
\hline No of Wireless node & 14 \\
\hline Simulation Time & 120 \\
\hline WiMAX(802.16) nodes & Small Yellow circle \\
\hline WLAN(802.11b) nodes & Big Brown circle \\
\hline
\end{tabular}

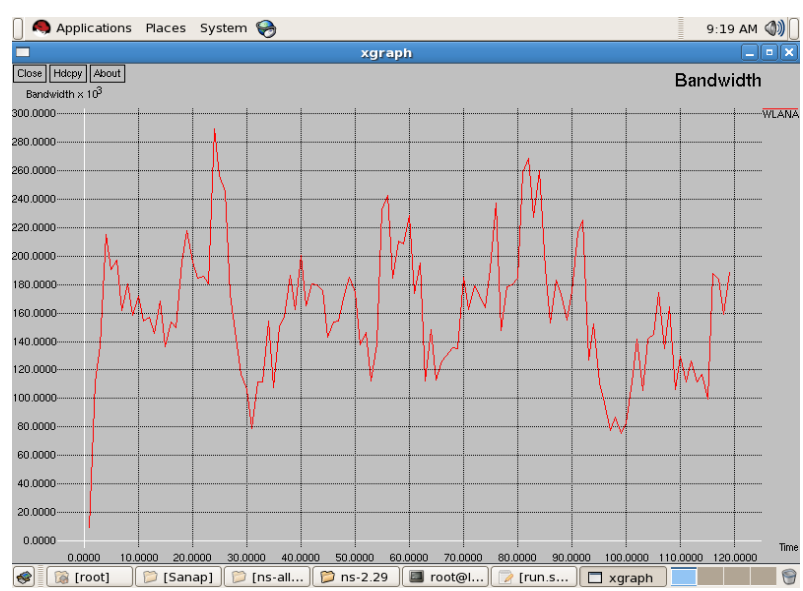

In this section discuss the simulation and results analysis of proposed work. For implementing this used network simulator version 2.29.In simulation, considered proposed integrated WLAN (802.11) and WiMAX (802.16) network. The result of network topology of work as shown in Fig 5. Random topology is created there are total 20 nodes some movable node and some are fix node of integrated network. Also created one AP and one BS. Nodes with small yellow circle indicate WiMAX nodes and nodes with big brown circle indicate WLAN nodes.
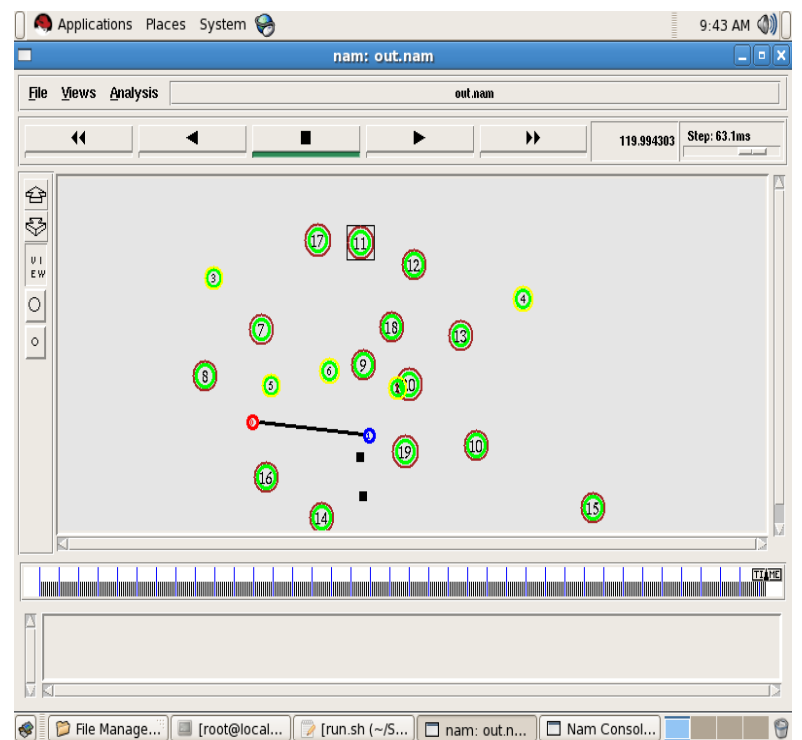

\section{Fig 5 Network topology}

The Fig 6 and Fig 7 show available bandwidth, utilized bandwidth of WLAN system. In handoff method is done based on available bandwidth, power consumption, security and cost of node which gives results in greater throughput. Relationship between available bandwidth and utilized bandwidth with respect to time is shown.

\section{Fig 6 WLAN_AB_New}

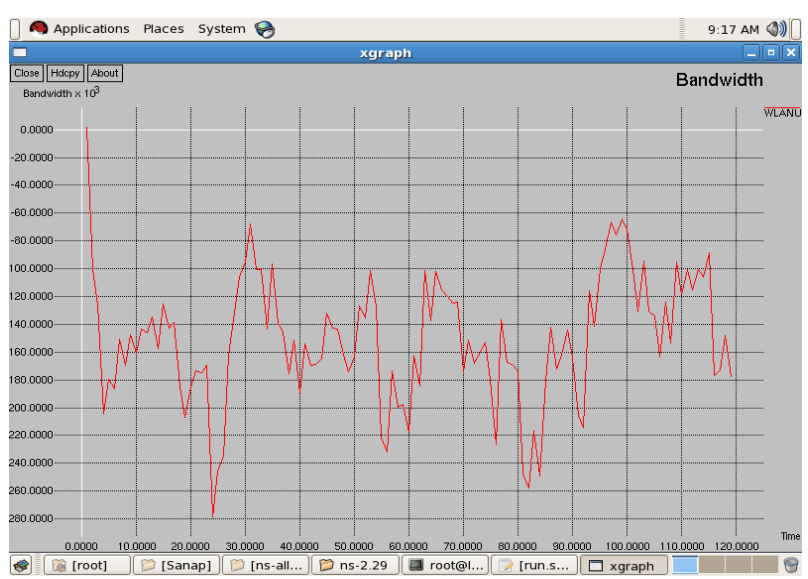

Fig 7 WLAN_UBNew

Following Fig 8 shows combine utilized and available bandwidth of WLAN with relationship between available and utilized bandwidth on $\mathrm{x}$-axis shown and time on $\mathrm{y}$-axis.

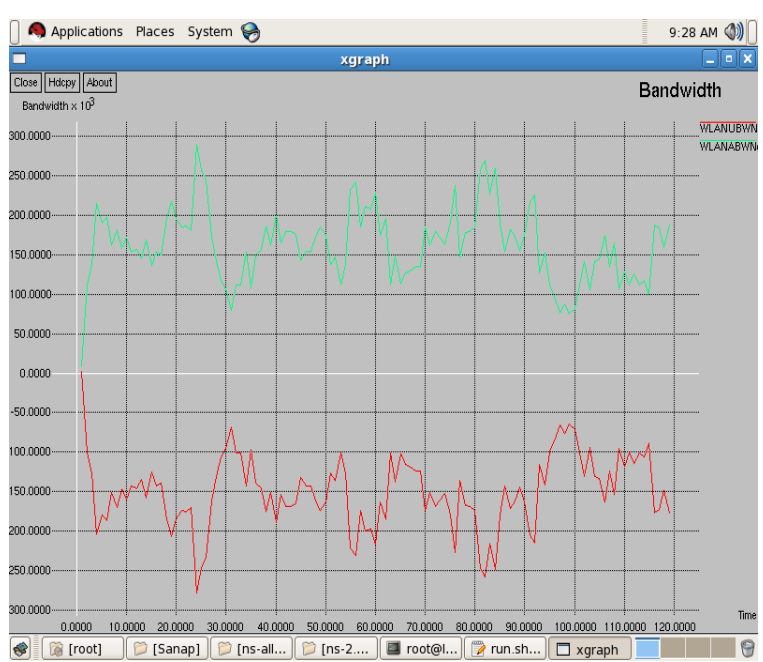

Fig 8 WLAN_UB_AB_New

Similarly, the Fig9 and Fig10 shows available uplink and utilized uplink bandwidth of WiMAX system and Fig 11 shows combine available uplink and utilized uplink bandwidth of WiMAX system. Relationship between 
available bandwidth and utilized bandwidth with respect to time is shown

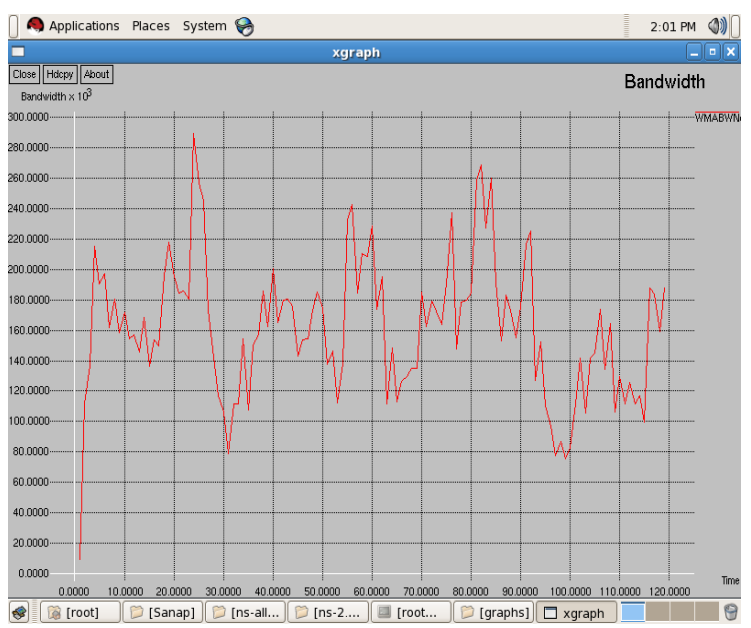

Fig 9 WiMAX_AB_Uplink_BW_New

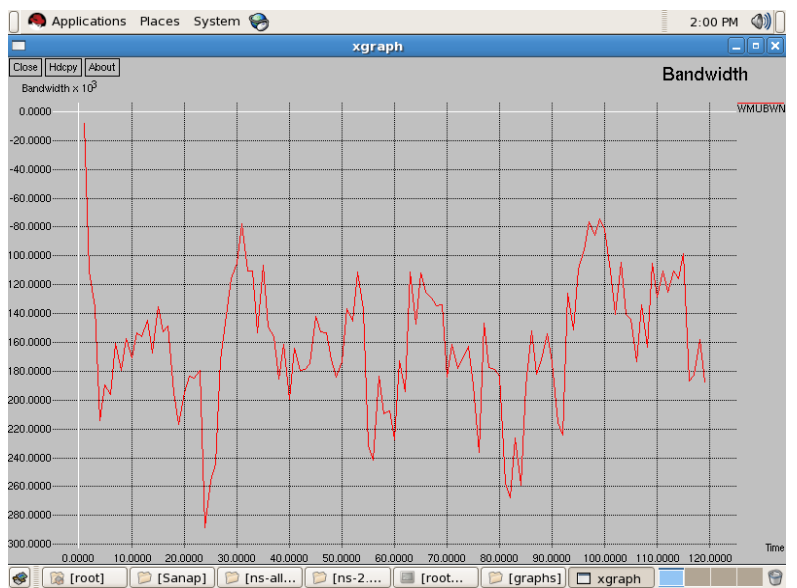

Fig 10 WiMAX_UT_Uplink_BW_New

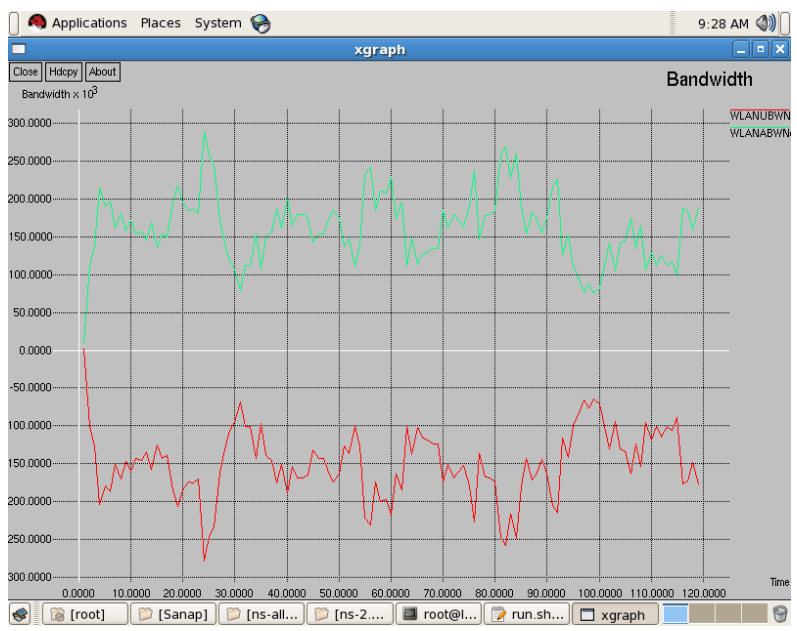

Fig 11 WiMAX_UB_AB_Uplink_New

Following Fig 12 shows energy consumption of proposed system with relationship between bandwidth on $\mathrm{x}$-axis shown to time on y-axis.

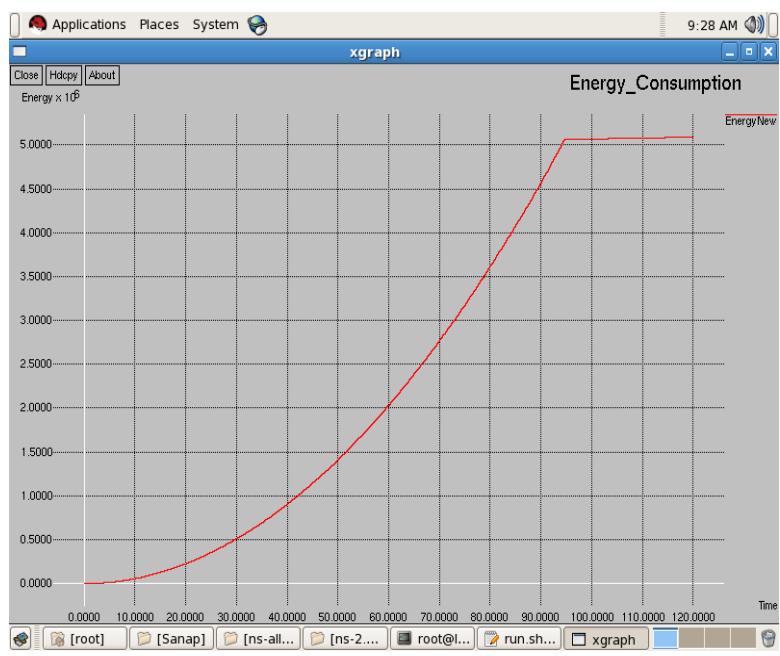

Fig 12 Energy_consumption

The Fig 13 shows throughput obtained in existing system. Initially, the station was served by WLAN service domain and gives better throughput. But after some second, VHOM detected that the network traffic tended to be overloaded and didn't get constant throughput. The NCDM module was initiated for estimate the available bandwidth of WiMAX. The estimated results showed that both the downlink and uplink available bandwidth of WiMAX could satisfy the requirements. Then the connections were transferred to the WiMAX domain immediately at the station. The throughput obtained at the station after estimating the bandwidth remain constant only for few seconds and again it decrease as the neighbouring node to whom connections are transferred is not having enough power. Then HDM again search for another network with available bandwidth, enough power of node. So in this graph can see there is no constant throughput.

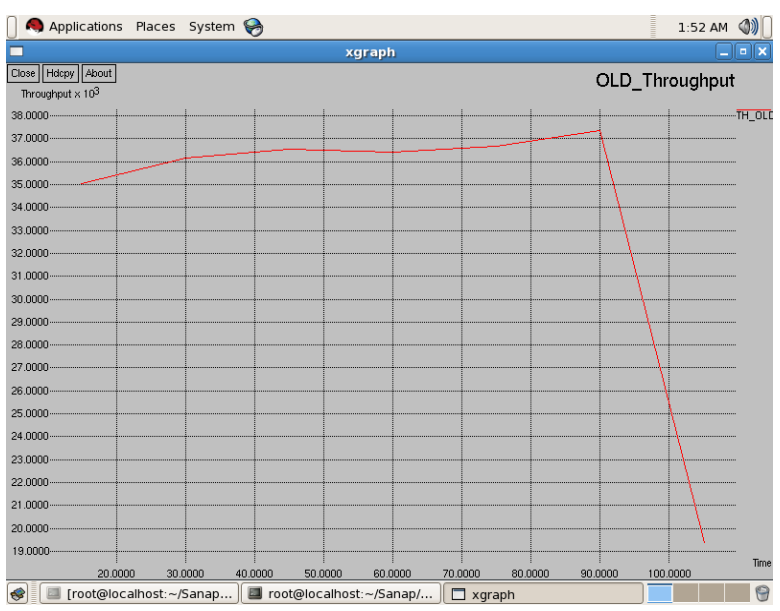

Fig 13 Throughput_Old

In Fig 14 the results show greater throughput can be obtained by the proposed solution. Because in proposed scheme after estimating available bandwidth search for neighboring nodes and calculate their power and select maximum power of node, it also check the security level of node, assign security level to every node randomly i.e. high level security (1) and other is low level of security ( 0 or none) from that select the higher level security node but if there is only one nodes available and there is no security at that situation select it, also in case of cost of node assign different cost to different nodes and from that HDM select minimum cost of node. In addition in 
security of node data will be encrypted and decrypted at sender as well as receiver side. So in proposed system there is constant throughput in graphs.

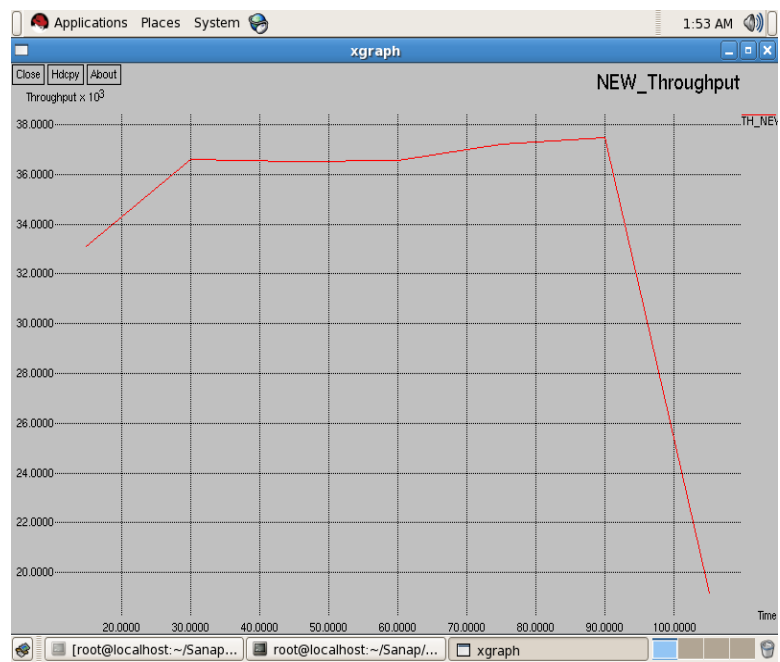

Fig 14 Throughput_New

Fig 15 shows the comparison of existing and proposed scheme from which come to know that better throughput achieved through proposed scheme.

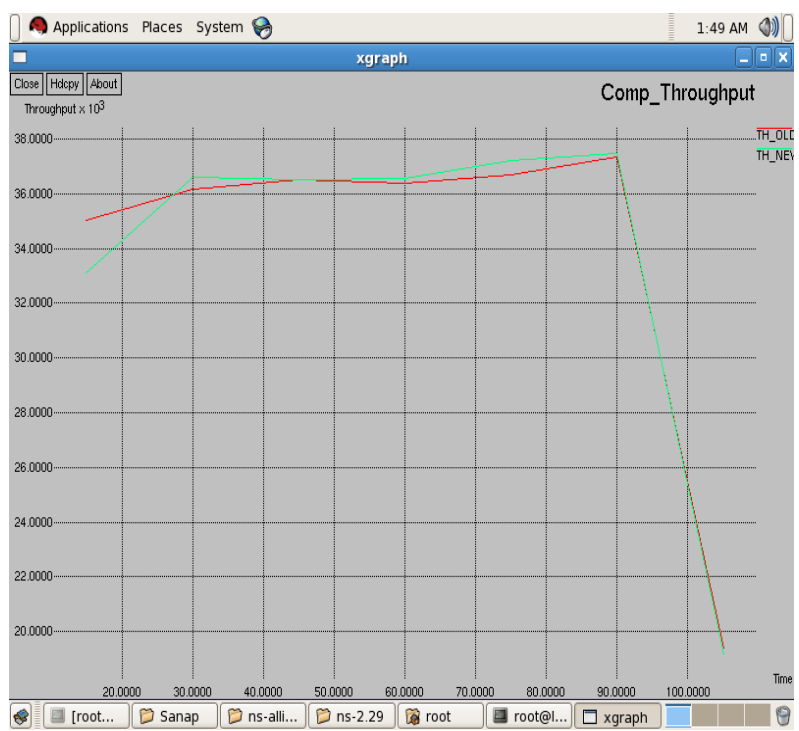

Fig 15 Throughput_Old_New

\section{CONCLUSION}

In this paper, proposed a novel VHOM scheme to make VHO decisions in the interworking architecture of WLANs and WiMAX networks. The scheme aims to provide always the best QoS in terms of throughput for both mobile users and fixed users. The available bandwidth power consumption, Network cost and Security of node has been taken into account in making the vertical handoff decisions. Two existing bandwidth algorithms is used to estimate the available bandwidth in WLANs and WiMAX networks respectively, which are based on the inherent features of the bandwidth allocation schemes and the message transmission approaches in both networks. As well as in proposed scheme calculate remaining power of neighboring node so that get better throughput, and in proposed scheme finds minimum cost of network and highest security level should be considered. The parameters required for the bandwidth evaluation and power consumption are easy to obtain in the real networks and the complexity of the evaluation is computationally low. Those make the proposed scheme to be feasible to implement and selecting proposed scheme is satisfy the fully QoS parameter during vertical handoff.

\section{FUTURE WORK}

The available bandwidth, power consumption, security and cost of node have been taken into account in making the vertical handoff decisions in proposed scheme. However, it is difficult to consider all the parameters during designing the decision model for VHO but if by considering more parameters in future work, the outcome of the decision mechanism would definitely improve.

In the future study can extend proposed scheme by considering another factor channel utilization as well as Handoff time optimization.

\section{REFERENCES}

[1] E. Gustafsson and A. Jonsson, "Always best connected," IEEE Wirel. Commun., vol. 10, no.1, pp. 49-55, Feb 2003.

[2] M. Liu, Z. Li, X. Guo, and E. Dutkiewicz, "Performance analysis and optimization of handoff algorithms in heterogeneous wireless networks", IEEE Trans. Mobile Comput., vol. 7, no. 7, pp. 846-857, July 2008.

[3] L. Wonjun, K. Eunkyo, K. Joongheon, L. Inkyu, and L. Choonhwa "Movement-aware vertical handoff of WLAN and mobile WiMAX for seamless ubiquitous access," IEEE Trans.Consum.Electr., vol. 53, pp. 1268-1275, 2007.

[4] J. J. J. Roy, V. Vaidehi, and S. Srikanth, "Always bestconnected QoS integration model for the WLAN WiMAX heterogeneous network", IEEE Int. Conf. on Industrial and Information Systems (ICIIS), pp. 361$366,2006$.

[5] J. Nie, J. C. Wen, D. Qi, and Z. Zhou, "A seamless handoff in IEEE 802.16a and IEEE 802.11n hybrid networks", IEEE Int. Conf. on Commun. Circuits and Systems, vol. 1, pp. 383-387, 2005.

[6] Valerie E. Zelenty; LAN, MAN Standard Committee of IEEE Computer Society; Wireless LAN Medium Access Control (MAC) and Physical Layer (PHY) Specification.

[7] IEEE 802.11 WG, Part 11: Wireless LAN medium access control (MAC) and physical layer (PHY) specifications, IEEE standard, 1999.

[8] IEEE 802.16 WG, Part 16: Air Interface for fixed broadband wireless access systems, IEEE Standard, 2004.

[9] N. Nasser, A. Hasswa and H. Hassanein, "Handoffs in fourth generation hetero-geneous net- works", IEEE Communi-cations Magazine, Vol. 44, No. 10, pp. 96103, Oct. 2006

[10] Bradley Mitchell; Wireless Standards 802.11b, 802.11a, 802.11gand802.11n,www.compnetworking.about.com/cs /wireless80211/a//aa80211standard.htm.

[11] Markus Kujala; WLAN Standards and Wireless Networking Security, Department of Telecommunication Software and Multimedia Laboratory, Helsinki University of Technology, May 28,2008.

[12] Marks, L. Victor, The 802.11g standard. IEEE IBM developer Works Wireless articles http://www 
106.ibm.com/developerworks /wireless /library/wiieee.html.

[13] M. Stemm and R. H. Katz,Vertical handoffs in wireless overlay networks, "Mobile Networks and Applications, vol. 3, no. 4, pp. 335-350, 1998.

[14] Pramod Goyal S. K. Saxena, (2008)A Dynamic Decision Model for Vertical Handoffs across Heterogeneous Wireless Networks,6772008 WASET.ORG, World Academy of Science, Engineering and Technology, Issue 41,pp 676-682.

[15] K.Ayyappan and P.Dananjayan, (2008) "RSS Measurement for Vertical Handoff in Hetrogeneous Network", International Journal of Theoretical and Applied Information Technology, Vol.4, No.10.
[16] Dong Ma and Maode Ma, A QoS-Based Vertical Handoff Scheme for Interworking of WLAN and WiMAX, IEEE “GLOBECOM” 2009.

[17] R. Prasad, C. Dovrolis, M. Murray, and K. Claffy, "Bandwidth estimation: metrics, measurement techniques, and tools", IEEE Network, vol. 17, no. 6, pp. 27-35, NOV.- DEC. 2003

[18] IEEE 802.11 WG, Part 11: Wireless LAN medium access control (MAC) and physical layer (PHY) specifications, IEEE standard, 1999.

[19] Bob Elliot, Elsa Mardrigal; Choosing the right WLAN Architecture,www.panduit.comhttp://www.panduit.com/ groups/MPMWC/documents/Article/107242.pdf, June 2006. 